QVIPURAMAYOC Revista de la Facultad de Ciencias Contables

Vol. 16 N³2, pp.105-115 (2009) UNMSM, Lima, Perú

ISSN: 1560-9103 (versión impresa) / ISSN: 1609-8196 (versión electrónica)

\title{
LA FACULTAD DE CIENCIAS CONTABLES DE LA UNIVERSIDAD NACIONAL MAYOR DE SAN MARCOS ANTE EL CIBER-PLAGIO
}

\author{
THE FACULTY OF COUNTABLE SCIENCES OF THE NATIONAL MAJOR \\ UNIVERSITY SAN MARCOS AGAINST THE CYBER-PLAGIARISM
}

\author{
Carlos Alberto Pastor Carrasco* \\ Docente Asociado de la Facultad de Ciencias Contables, UNMSM
}

[Recepción: Abril de 2009 / Conformidad: Mayo de 2009]

\section{RESUMEN}

El presente artículo se propone examinar el plagio en general, en su doble vertiente: una por la apropiación ilícita (contra el autor de la obra plagiada) y otra por el fraude realizado (respecto de los destinatarios de la obra realizada con plagio). Con estas premisas se analiza el ciber-plagio académico definido como una forma de plagio realizada con la ayuda de herramientas electrónicas disponibles en el ámbito universitario.

Además se pretende: a) Explorar la naturaleza del plagio on-line; b) Describir modelos para prevenir el plagio; c) Presentar tecnología que puede utilizarse para identificar el plagio; y d) Explorar formas efectivas de responder al plagio, así como el tratamiento disciplinario del ciber-plagio con la normativa de que disponen actualmente las universidades.

Palabras clave: Plagio, ciber-plagio, régimen disciplinario, reglamento académico.

\begin{abstract}
The present article intends to browse the abduction in general, in its pouring double: one for the fraudulent conversion and (against the author of the pirated work) another for the realized (regarding the addressees of the realized work with abduction) fraud. With these premises the academic defined ciber-plagio is analyzed as an abduction form carried out with the help of electronic available tools in the university environment. It is also sought: a) to Scan the nature of the online abduction; b) to Describe models to prevent the abduction; c) to Enter technology that can be used to identify the abduction; and d) to Scan actual forms of responding to the abduction, as well as the disciplinary treatment of the ciber-plagio with the regulatory scheme that they preparate the universities currently.
\end{abstract}

Keyword: Pirate, ciber-plagio, régime disciplinarian, academic regulation.

* Contador Público Colegiado Certificado. E- mail: cpc5637@hotmail.com 
De todos los animales, el hombre es el único que miente. Mark TwaIN

(1835-1910)

\section{INTRODUCCIÓN}

En la Conferencia Mundial sobre la Educación Superior: La educación superior en el siglo xxi: Visión y acción, llevada a cabo en Francia, se emitió la Declaración Mundial sobre la Educación Superior en el Siglo XXI: Visión y acción, en la que en su artículo 12 señalaó lo siguiente sobre el potencial y los desafíos de la tecnología:

"Los rápidos progresos de las nuevas tecnologías de la información y la comunicación seguirán modificando la forma de elaboración, adquisición y transmisión de los conocimientos. También es importante señalar que las nuevas tecnologías brindan posibilidades de renovar el contenido de los cursos y los métodos pedagógicos, y de ampliar el acceso a la educación superior. No hay que olvidar, sin embargo, que la nueva tecnología de la información no hace que los docentes dejen de ser indispensables, sino que modifica su papel en relación con el proceso de aprendizaje, y que el diálogo permanente que transforma la información en conocimiento y comprensión pasa a ser fundamental."

Por ello, para la comunidad universitaria, no ha pasado desapercibido que en la actualidad existe un gran avance en el terreno de las Tecnologías de Información y Comunicaciones (TIC), y en especial una espectacular popularización y crecimiento de la red Internet $\left(w w w^{1}\right)$. Son precisamente estos dos componentes los principales hechos que han motivado que se denomine a la actual sociedad como la "sociedad de la información" (SI).
Los estudiantes universitarios tienen ahora la posibilidad de acceder a ingentes cantidades de datos, información y conocimiento; esto les permiten seguir de manera casi instantánea los acontecimientos que ocurren en cualquier lugar del mundo, o también de disponer de nuevas maneras de ocio, o bien, documentarse a fondo sobre cualquier tema de su interés. Estos avances han traído aspectos positivos en el desarrollo de los trabajos de investigación de los alumnos universitarios, ya que se tiene acceso a grandes volúmenes de información, pero ahora es preciso disponer de un amplio criterio y habilidad para seleccionarla y saberla utilizar adecuadamente.

Asimismo, las TIC han provocado o facilitado algunos cambios de actitudes en los alumnos que no pueden valorarse en forma positiva. Es el caso del denominado "ciberplagio académico", mediante el cual los alumnos adoptan y presentan en sus trabajos de investigación como ideas propias, teorías e hipótesis realizados por otros investigadores, y son estas tecnologías asociadas a la Sociedad de la Información, las que facilitan esta práctica éticamente reprobable y académicamente incorrecta.

El plagio se ha convertido en una constante de nuestra sociedad. Pues no sólo ha logrado invadir el mundo académico, sino que también ha incursionado en otros espacios tales como la música, la pintura, el cine, etc., es decir, las ciencias y artes en general. Grandes escritores y artistas se han visto envueltos en escándalos por causa de este fenómeno. Esta práctica es tan antigua como la historia misma y lo curioso es que tan famoso "delito" se ha venido propagando, desde siglos atrás, con nuevas y sutiles modalidades.

Para los docentes representa un reto evitar que esta situación se propague exponen-

1 World Wide Web. 
cialmente entre los alumnos universitarios, por eso consideramos que es un tema prioritario para que nuestras autoridades establezcan la normatividad a seguir en el futuro inmediato.

\section{ANTECEDENTES}

Ahora bien, es cierto que el plagio siempre ha existido dentro del ámbito académico estudiantil; sin embargo, estudios y análisis que sobre el tema señalan que por efecto de un aumento exponencial de penetración de Internet; la mayor facilidad de acceso a los contenidos digitales en general y los albergados en la red en particular, han provocado un auge inusitado en las prácticas de plagio entre el alumnado universitario. Asimismo, se ha cambiado la manera de cómo se comete el plagio (evidentemente a través de recursos y contenidos de Internet) y, por otro lado, las facilidades con que disponen los alumnos a la hora de plagiar. El problema acaso sea el mismo que ha existido desde hace mucho tiempo, pero las circunstancias, la prevalencia y sobre todo sus repercusiones y profundidad son nuevas y parecen más peligrosas de lo que fueron en el pasado.
En la actualidad, la web se ha convertido en una fuente de referencia indispensable para la generación de documentación académica por quienes forman parte de cualquier proceso de enseñanza-aprendizaje, tanto para docentes como para los alumnos. En el Cuadro 1 Crecimiento de Servidores WWW 1996-2000, se observa dicho crecimiento.

La gran cantidad de información existente, tales como, materiales, artículos, multimedia, libros, revistas especializadas, bases de datos, portales temáticos, etc. disponibles a través de Internet -inclusive la mayoría de ellos no se puede acceder desde otro espacio o canal-, la facilidad y comodidad de acceso a los mismos, se puede acceder a cualquier hora, (situación que no ofrece nuestra biblioteca, ya que tiene un horario restringido y al cual no pueden acceder nuestros alumnos debido a que la mayoría de ellos trabajan durante el día y en la noche se encuentra en clases) y teniendo en cuenta que la mayor parte de ellos son gratuitos; son factores de gran ayuda para cualquier alumno universitario que desee consultar información con fines académicos $y$, en cierta manera, es inconcebible pensar que hoy se realice un trabajo académico, el planteamiento de una investigación, la ampliación de contenidos de una

GRÁFICO 1. CRECIMIENTO DE SERVIDORES WWW 1996-2000

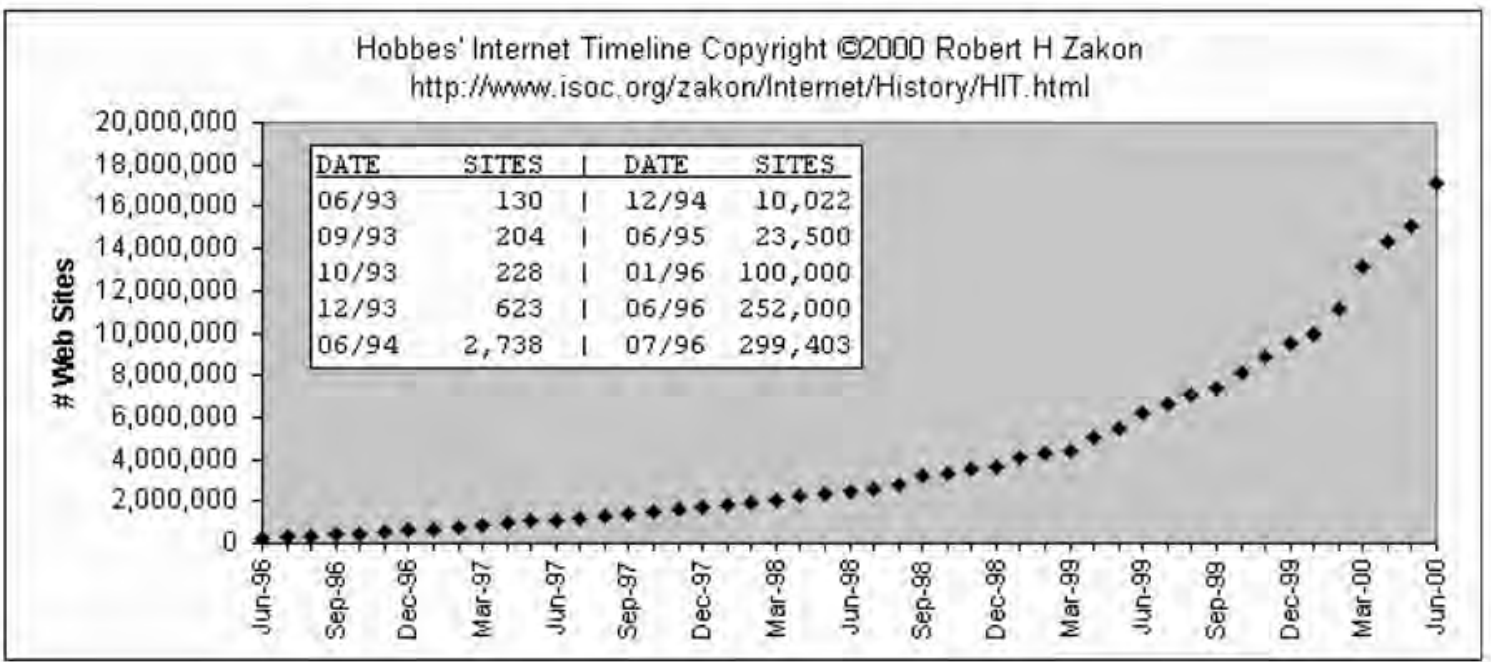


asignatura, el trabajo en un aula, etc. sin contar con la ayuda de Internet como elemento básico de consulta.

Sin embargo, esta irrupción de las Tecnologías de la Información y Comunicación ha provocado o facilitado algunos cambios de actitudes en los alumnos que no pueden valorarse necesariamente en forma positiva. Es el caso del denominado "ciber-plagio académico". Mediante el los alumnos adoptan y presentan en sus trabajos de investigación como ideas propias, teorías e hipótesis realizadas por otros investigadores, $y$ es precisamente estas tecnologías asociadas a la Sociedad de la Información (SI), sobre todo Internet y más concretamente la WWW, las que facilitan esta práctica académicamente incorrecta y éticamente reprobable. Este fenómeno se ha extendido tanto entre los estudiantes que algunos autores hablan de la existencia de una "Generación Copiar-yPegar- (Comas, Sureda \& Urbina, 2005). En la figura Ilustración 1 Teclas más empleadas se muestra las teclas más empleadas entre los estudiantes universitarios.

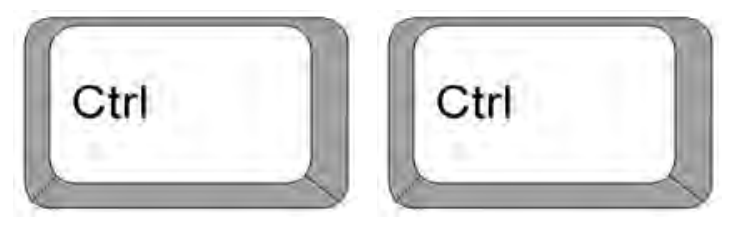

Ilustración 1. Teclas más empleadas.

Jason en un estudio llevado a cabo en el año 2006 determinó que "Actualmente la mayoría de los estudiantes que entran a la educación superior son más jóvenes que el microcomputador, y se sienten mejor escribiendo con el teclado que haciéndolo en un cuaderno de espiral, también prefieren leer en la pantalla del computador que hacerlo de papeles que sostienen con sus manos. La conectividad constante, estar en contacto permanente con amigos y familia, en cual- quier momento y desde cualquier lugar, es para ellos de la mayor importancia (Jason, 2006)."

La denominación de "generación de copiar y pegar" se ha popularizado como compendio de vía rápida para referirse a una generación cuyo primer punto de contacto con el conocimiento es Internet y un motor de búsqueda, Google es el más popular de estos últimos. Esto contrasta con nuestra generación que adquiríamos conocimiento mediante libros y el acceso a las bibliotecas convencionales.

Cabe mencionar que existen estudios y literatura asociada al concepto de ciber-plagio académico en los últimos años, desarrollados principalmente en países anglosajones; en otros contextos culturales se ha iniciado de manera cuasi exploratoria a trabajar en este ámbito. No se ha encontrado estudios que puedan aportar datos referentes al contexto latinoamericano, y sólo encontramos algunos artículos pioneros de españoles como los trabajos de Cabanillas (Cabanillas, 2008) y Urbina (Urbina, 2004).

\section{¿QUÉ ES EL PLAGIO?}

Empezaremos definiendo lo que se entiende por plagio según la definición dada por la Real Academia Española: Plagio (Del lat. plagǐum).

1. Acción y efecto de plagiar (II copiar obras ajenas).

2. Acción y efecto de plagiar (II secuestrar a alguien).

Por ello es necesario buscar la definición de plagiar, y encontramos: Plagiar. (Del lat. plagiāre).

1. tr. Copiar en lo sustancial obras ajenas, dándolas como propias. 
Se sostiene con mucho tino que "el plagio constituye el más grave atentado al derecho de autor, pues en esencia significa desconocer la paternidad del autor, y por consiguiente, la relación que le une con la obra sustrayéndole a todo conocimiento e ignorándole toda aportación creativa"(La Torre, 1994)

Es así que el delito de plagio atenta contra los derechos fundamentales que dimanan de la creación de una obra. Lesiona las facultades morales del autor sobre su creación, al tiempo que perjudica también los derechos de explotación. Del mismo modo, el delito de plagio atenta contra el interés público en sus diversas facetas en la medida en que la obra plagiada, por no ser original, engaña al consumidor con la suplantación se pierde el vínculo que existe entre el verdadero autor y el fruto de su espíritu creador.

De ahí la importancia de la represión penal del plagio. Los bienes jurídicos que protege hacen indispensable su tratamiento mediante la vía penal.

En los cursos de la universidad, estamos continuamente comprometidos con las ideas de las otras personas: los leemos en libros, los escuchamos en las conferencias, hablamos de ellos en la clase, y los incluimos en nuestro propio material escrito. En consecuencia, es muy importante que demos el crédito a los autores originales cuando corresponda. El plagio está usando las ideas y palabras de otros sin reconocer el origen de esa información.

En el ámbito académico, podemos distinguir dos tipos de plagio: El deliberado (intencional), y el inconsciente (por falta de conocimiento de lo que constituye plagio).

Definimos al plagio como deliberado o intencional cuando se realiza alguna de las siguientes acciones:

- Se compra, se roba o se toma prestado un trabajo redactado por otra persona para hacerlo pasar como propio;
- Se paga a otra persona para que le escriba el trabajo que posteriormente lo hará pasar como propio; o

- Cuando se copian adrede las palabras o ideas de otros, sin darle crédito, para hacerlas pasar como propias

Finalmente, decimos que puede ocurrir un plagio "accidental" cuando sucede lo siguiente:

- La persona no sabe citar correctamente,

- Se parafrasea sin realmente alejarse del texto original; o

- Cuando se desarrollan pensamientos o teorías basadas en ideas ajenas, sin dar crédito a la persona sobre cuya idea se basa la discusión.

\section{DOBLE DELITO: APROPIACIÓN ILÍCITA Y FRAUDE}

La Constitución del Perú de 1993, en su artículo 2 inc. 8., determina como derechos de la persona: "A la libertad de creación intelectual, artística, técnica y científica, así como a la propiedad sobre dichas creaciones y a su producto. El Estado propicia el acceso a la cultura y fomenta su desarrollo y difusión."

Por tal motivo el acto de plagiar supone cometer dos delitos. "Robar al autor y engañar al destinatario de la obra plagiada”. Es decir un acto de plagio afecta simultáneamente a dos grupos de intereses:

- Los intereses del autor (y, en su caso, el del titular de los derechos de explotación de la obra, como es, por ejemplo, el editor);

- Los intereses del destinatario de la obra, a quien se pretende engañar haciéndola pasar como propia.

El plagio no sólo perjudica al autor de la obra plagiada sino que también es un fraude 
que perjudica a su destinatario. Independientemente de que el fraude se realice a costa del autor, lo cierto es que el perjuicio de éste es instrumental, un mal necesario para lograr el objetivo principal, que es el engaño del destinatario de la obra plagiaria.

En el campo del derecho público, el engaño puede perseguir que el plagiario aparente mayores méritos de los que posee, al atribuirse la autoría de la obra ajena o de parte de ella. De esta manera, puede, por ejemplo, cumplir los requisitos para obtener una licencia o autorización, aprobar un examen, ganar un concurso, etc.

En algunos casos (en particular, concursos), puede verse afectado, además, de un interés público, el de los candidatos concurrentes que se ven preteridos gracias a los falsos méritos presentados por el plagiario.

En este trabajo de investigación nos vamos a ocupar únicamente de la segunda cara del plagio, la que supone un fraude para el destinatario del trabajo, es decir, a los docentes de la Facultad de Ciencias Contables o a sus autoridades.

¿Por qué debe ser sancionado el plagio en la Facultad?

¿Por qué el acto de plagiar debe ser considerado como una falta grave? Se podrían mencionar tres razones para sancionar el plagio en la Facultad en el campo específico del trabajo universitario.

La primera razón y la más importante consiste en entender que el plagio es equivalente a negarse a utilizar el derecho de pensar por sí mismo. En efecto, cuando los alumnos realizan un trabajo de investigación o preparan una exposición oral y utilizan como propias las ideas de otros autores, están emplean- do ideas que otros pensaron antes por lo que no tienen necesidad de pensar por sí mismos. Según los objetivos de la Universidad ${ }^{1}$, la tarea principal que deben realizar docentes y alumnos es pensar. Si se plagia, se está haciendo algo contradictorio con la esencia de la Universidad y están degradando la calidad que buscan los demás profesores y alumnos. En otras palabras, se está traicionando al esfuerzo de la comunidad universitaria.

La segunda razón tiene que ver con otro mandato dado en la Ley Universitaria ${ }^{2}$, ya que si partimos del principio de que cuando se plagia no se piensa, entonces se retrasa el progreso del conocimiento humano, porque, a pesar de que tengamos la posibilidad de hacer un trabajo creativo en la Universidad (oportunidad que pocos tienen en la vida), no se cumple con esa responsabilidad. Los alumnos tienen el deber de hacer su mejor esfuerzo por pensar, en beneficio de la $\mathrm{Hu}$ manidad, ya sea que se logre grandes resultados o no.

Finalmente, la última razón considera un aspecto fundamental que tiene el plagio, hay que recordar que existe un comportamiento contrario a la ética, tanto porque se incumple con el deber de trabajar como universitarios como porque, al tomar las ideas de otros y hacerlas pasar por nuestras, se las estamos robando. El plagio es por ello una forma de hurto, no tiene ninguna excusa hacerlo, y no debe ser permitido o tolerable en ningún nivel de la universidad. Lo ético es cumplir bien con los deberes y reconocer a cada uno lo que es suyo, especialmente si es su creación. Por otro lado, al presentar un trabajo ajeno como propio, están distorsionando la evaluación que le corresponde hacer a los docentes.

1 Ley 2733 Artículo $1^{\circ}$ - "Las Universidades están integradas por profesores, estudiantes y graduados. Se dedican al estudio, la investigación, la educación y la difusión del saber y la cultura, y a su extensión y proyección sociales. ...”

2 Ley Universitaria $\mathrm{N}^{\circ} 23733$, Artículo 2 c) "Formar humanistas, científicos y profesionales de alta calidad académica, de acuerdo con las necesidades del país, desarrollar en sus miembros los valores éticos y cívicos ...” 


\section{¿EXISTEN RAZONES PARA PLAGIAR?}

En una encuesta rápida, realizada entre los alumnos del 5to año de la Facultad de Contabilidad, el año pasado, se determinó que las razones que los impulsaban a plagiar podrían consolidarse en cuatro:

- Escasez de tiempo.

- Algunos consideraban que como el curso asignado en el Programa académico era una pérdida de tiempo y que no aportaba nada a su desarrollo profesional no deberían dedicarle tiempo en el desarrollo de los trabajos.

- En razón que los alumnos de los últimos años, además de estudiar, deben realizar sus prácticas pre-profesionales no les dejaba suficiente tiempo libre para el desarrollo de sus trabajos de investigación.

- Algunos reconocían que les faltaba organizarse para asignar tiempo adecuado a cada una de las materias de la carrera.

- Razones relacionadas con la ética y la sociedad.

- El plagio se ha convertido en una práctica social aceptada.

- No se tienen claras las metas de la educación, en el sentido de que están en la universidad para aprender a aprender y no para aprobar cursos.

- Resistencia de algunos profesores a tomar una acción correctiva drástica a fin de evitar su reincidencia desde los primeros años de su formación.

- Sobrevivencia.

- Debido a que existe competencia para conseguir trabajo y para seguir cursos de post-grado.

- Finalmente, se indicó que: "Todo el mundo lo está haciendo"
- Conocimiento.

- Los alumnos no han desarrollado eficientemente sus destrezas de investigación.

- Asimismo no tienen destrezas para efectuar citas, por no haber recibido instrucciones específicas sobre el particular.

- No entienden el curso ni el objeto del trabajo asignado.

- No entienden la naturaleza del plagio y lo que significa el respeto de los derechos de autor.

- Algunos demostraron falta de confianza en sus capacidades cognoscitivas.

Como se podrá apreciar, motivos para realizar el plagio no les faltaban.

En la actualidad existen varias páginas web que han sido específicamente diseñadas para que los estudiantes universitarios puedan hacer búsquedas por tema y escoger los trabajos que más se asemejen a las tareas asignadas por sus docentes.

Una vez identificado el ensayo que se asemeje a la tarea asignada, proceden a imprimirlo y a entregarlo con su nombre, como si se tratase de un trabajo original.

Dentro de las páginas web existentes en español se puede mencionarse a las siguientes:

- http://www.monografias.com/

- http://www1.rincondelvago.com/apuntes.html

- http://www.alipso.com/

Sin embargo, las páginas web disponibles en inglés son mucho más numerosas:

- http://www.schoolsucks.com/

- http://www.cyberessays.com/

- http://www.planetpapers.com/

- http://www.netessays.net/

- http://www.essaydepot.com/ 
- http://www.cheathouse.com/

- http://www.a1-termpaper.com/

- http://www.cheater.com/

- http://www.freeessay.com/

- http://www.007termpapers.com/

\section{MODELOS PARA EVITAR EL PLAGIO}

Los modelos para evitar el plagio podría reducirse a dos: el de la prevención, a fin de evitar que el plagio suceda en la universidad y el de la mano dura, es decir, castigar a los que la realizan. Nosotros nos inclinamos por el primero porque para aplicar el segundo modelo sería más difícil de detectar en algunas circunstancias.

Una combinación de ambos modelos lo viene realizando la Pontifica Universidad Católica del Perú, la que en su página web ha incluido un link denominado "Bienvenida la Integridad" en la cual se muestran afiches como el que se muestra en la Ilustración 1 PUCP-Integridad.

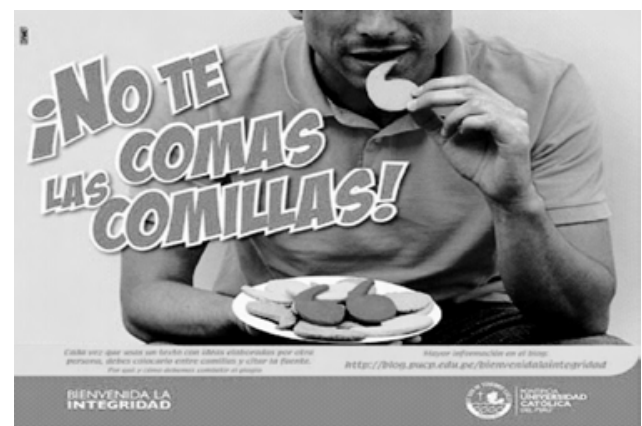

Ilustración 2. PUCP-Integridad

Por otro lado, ha incluido en su reglamento Disciplinario el Artículo 4 Infracciones Graves, inciso c) "Cometer plagio o cualquier otro acto que intente alterar o distorsionar la objetividad de la evaluación académica...”. Haciéndose acreedores a lo siguiente: "Quienes incurran en infracciones de mayor gravedad, contempladas en el artí- culo $4^{\circ}$ del presente reglamento, podrán ser sancionados con suspensión académica hasta por veinticuatro meses o con expulsión de la Universidad.”

\section{ESTRATEGIAS PARA EVITAR EL PLAGIO}

Dentro de las estrategias que podrían elaborarse para evitar que los alumnos cometan plagio podríamos indicar a las siguientes:

- Proveer instrucciones específicas en torno al tema del trabajo escrito que se le ha requerido al estudiante, pues ello dificultará el que puedan entregar cualquier ensayo o artículo ya existente en la Internet.

- Seleccionar tareas y eventos únicos y específicos sobre los cuales exista muy poca información.

- Utilizar temas que integren teoría y experiencias personales de cada alumno.

- Minimizar el número de tareas, preferible es que sean de calidad a tener cantidad.

- Proveer una lista de tópicos específicos para los trabajos y cambiarlos todos los años.

- Las fuentes bibliográficas deben ser recientes.

- Trabajos en las que deban de realizar entrevistas y encuestas en las empresas.

- También es importante requerir el trabajo por etapas (Primer borrador, segundo borrador, trabajo final).

- Requerir presentaciones orales y bibliografías anotadas.

- Requerir a los estudiantes las fotocopias de las lecturas que utilizaron para el proyecto.

- Pedir bibliografías anotadas antes de la fecha límite.

- Incluir preguntas como: ¿Qué aprendió del trabajo? ¿Cuáles fueron los obstáculos que encontró? 
- Visitar estas páginas ocasionalmente para que se familiarice con su contenido y pueda identificar con facilidad los trabajos que sean producto de este tipo de páginas.

- No estaría de más decir a los estudiantes que se conoce las páginas web en donde se consiguen asignaciones y ensayos ya preparados. Esto disminuirá grandemente la probabilidad de que utilicen artículos y ensayos de estos sitios para su clase.

- Visitar alguna de estas páginas web con sus estudiantes durante una de sus clases, seleccione uno de los artículos allí disponibles y proceda a demostrar todo lo que está incorrecto con el mismo. Si los estudiantes entienden que estos trabajos son de mala calidad, se disminuye la probabilidad de que los utilicen.

- Ayudar a los estudiantes a entender el valor de lo que están aprendiendo.

- Recalcar y recompensar la originalidad.

- Debe fomentarse el respeto entre estudiantes/profesores.

- Estimular el amor por aprender y no sólo por aprobar.

- Fomentar un ambiente de confianza en la sala de clases.

- Estimular la responsabilidad del estudiante.

- Evitar asignaciones en la que sólo se le piden a los alumnos obtener datos y describir conceptos ya que ellas por sí misma facilitan el plagio.

En los casos de trabajos de investigación que deban realizarse en grupos de alumnos, debe especificarse las siguientes normas:

- Un trabajo en grupo debe ser una tarea que se inicia con la programación conjunta inicial, prosigue con el reparto de tareas que deben ser asumidas responsa- blemente y culmina con la puesta en común antes de su elaboración final.

- Un trabajo en grupo no es un trabajo hecho por partes que después se juntan para su entrega final.

- Para que el trabajo en grupo realmente cumpla con los objetivos señalados más arriba, debe haber un constante intercambio de los hallazgos realizados individualmente, de modo que todo el grupo no pierda de vista el conjunto del trabajo.

- El trabajo en grupo implica un trabajo solidario en el sentido de que el grupo en su conjunto asume los logros y errores del producto final. Esto no implica que se deba asumir y "tapar" a los alumnos que no realicen la parte individual que les ha correspondido, recargando con ello el trabajo de los alumnos responsables.

- Si alguien del grupo no trabaja como es debido, esto deberá ser oportunamente informado - esto es, en el momento de la presentación del trabajo y antes de que haya sido calificado- al profesor correspondiente.

- Una vez calificado un trabajo grupal, su calificación se asume de forma grupal y no habrá calificaciones diferenciadas a cada miembro del grupo.

- Si el profesor del curso detecta al momento de calificar que en el trabajo grupal se ha incurrido en plagio, el trabajo será desaprobado para todos los miembros del grupo por igual y la responsabilidad del hecho también se asume como grupal.

\section{TECNOLOGÍAS PARA DETECTAR EL PLAGIO}

Si se sospecha que algún estudiante ha entregado un trabajo que no es de su autoría, 


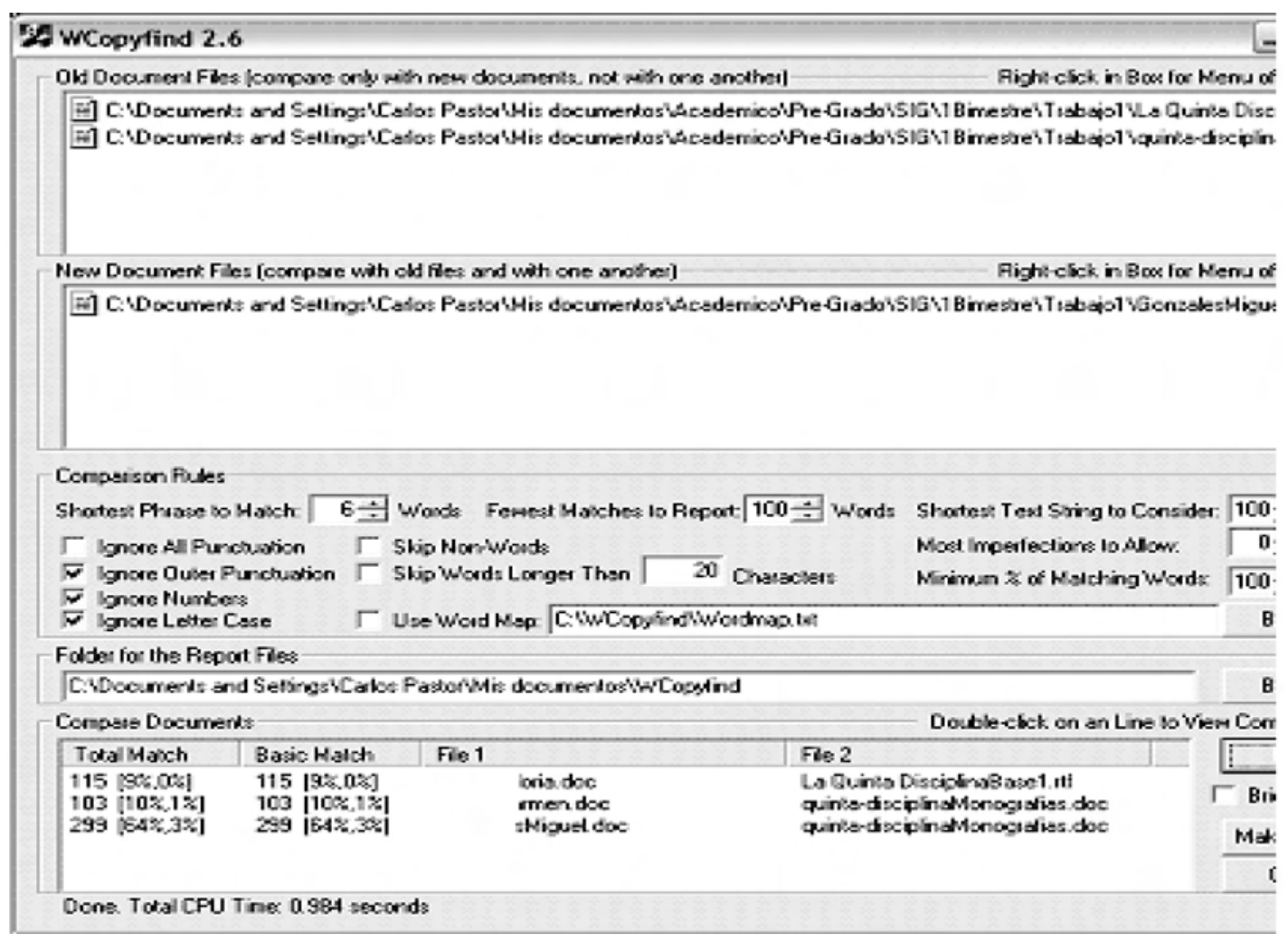

existen páginas en la Internet e incluso programas que ayudan a identificar los trabajos plagiados.

Los programas antiplagio permiten comparar trabajos de estudiantes entre ellos mismos y los comparan también con artículos disponibles en línea. Tienen disponible un "free trial" de un mes de duración.

Existe el software gratuito denominado WCopyfind, el mismo que esta disponible en Internet, que presenta el porcentaje de copia que ha realizado de los documentos originales.

A los interesados en obtener información adicional en torno a cómo funciona el software Turnitin, pueden visitar la siguiente página: http://www.plagiarism.org/technology.html

Finalmente, también el uso de motores de búsqueda como el de Google, puede ser efectivo y además es gratuito.

\section{RECOMENDACIONES}

1. Debe incluirse dentro del Plan de Estudios de todas las Escuelas Académicas de la Facultad de Ciencias Contables, el curso de Redacción y Argumentación, en él debe realizarse la presentación de un trabajo final. En la elaboración de dicho trabajo final se pondrá especial interés en enseñar a los alumnos cómo citar y lo importante de respetar la autoría intelectual de las fuentes.

2. Creación de un consejo de honor donde se evalúe el trabajo intelectual de los docente y alumnos

3. Desarrollo de tutoriales sobre honestidad académica, colocados en la página web de la Universidad y de la Facultad.

4. Revisión del Reglamento Estudiantil de la Facultad a fin de elevar recomendaciones a las autoridades para uniformizar criterios con otras facultades, 
donde se podría incluir entre otras a las siguientes:

a) Amonestación

b) Alumno en observación por tiempo definido durante el cual otra violación de cualquier norma tendrá consecuencia de suspensión o separación.

c) Suspensión de la Universidad por un tiempo definido.

d) Separación definitiva de la Universidad.

5. Facilitar el desarrollo de una cultura que fomente la honestidad académica

\section{CONCLUSIONES}

1. Cuando el alumno realiza el plagio, está revelando que no puede aprender, que no quiere aprender o que no le interesa aprender. En cualquiera de los casos, es una señal de peligro que emite el alumno y por ello requiere nuestra atención e intervención inmediata.

2. El plagio realizado por los estudiantes nos deja apreciar que algo no está bien en la relación profesor-estudiante y por ello debemos estar atentos para mejorarla.

3. La ejecución del plagio se convierte en un acto de infidelidad hacia el aprendizaje. Los que plagian no están amando el aprender, le están siendo infieles. Más allá de ser una falta de respeto para el profesor, es una profanación al acto sagrado del aprender. Plagiar es una negación del yo, es un acto de inautenticidad.

4. Realizar plagio dentro de la Universidad es faltar a los objetivos prioritarios establecidos en la Constitución y la Ley Universitaria y en nuestro estatuto, es fallarle al país entero y la comunidad universitaria en particular.

\section{REFERENCIAS}

1. Cavanillas, Santiago (2008). «El ciberplagio en la normativa universitaria». En: R. COMAS, J. SUREDA (coords.). El ciberplagi acadèmic [dossier en línea]. Digithum. No. 10. UOC. [Fecha de consulta: 31/03/09]. Accesible desde http://www.uoc.edu/digithum/10/dt/ esp/cavanillas.pdf

2. Comas, Sureda \& Urbina (2005): The "Copy and Paste" Generation: Plagiarism amongst Students, a Review of Existing Literatura. Internacional Journal of learning. Volumen 12.

3. Comas, Rubén \& Sureda, Jaume, 2007, Ciber-Plagio Académico. Una aproximación al estado de los conocimientos, Revista TEXTOS de la CiberSociedad, 10. Temática Variada. [Fecha de consulta: 02/04/09]. Disponible en http://www. cibersociedad.net

4. Decreto Legislativo 822 - Ley sobre el derecho de autor del 23 de abril de 1996.

5. Jason Frand, (2006). "The information mindset: Changes in students and implications for higher education", EDUCAUSE Review, March / April, p.15.

6. La Torre, V. (1994). Protección penal del derecho de autor. España: Ed. Tirant lo Blanch.p. 175

7. Urbina Ramírez, S. (2004): "Ciberplagio: 'construyendo' trabajos universitarios." EDUTEC 2004. [Fecha de consulta: 10/04/09]. Accesible desde http:// edutec2004.lmi.ub.es/pdf/159.pdf.

Vol. 16 (1) 2009 | QUIPURAMAYOC / 115 